Acta Hispanica (2020) Supplementum II: 737-746

\title{
RELACIONANDO EL PATRIMONIO CULTURAL MATERIAL E INMATERIAL PARA SU USO Y PROTECCIÓN EN LA SIERRA NORTE DEL PERÚ
}

\author{
YUJI SEKI \\ Museo Nacional de Etnología, Japón \\ Daniel SaUcedo Segami \\ Universidad de Ritsumeikan, Japón
}

\begin{abstract}
Resumen: Recientemente, el Perú ha tomado una actitud positiva en cuanto a la puesta en valor del patrimonio cultural como un elemento importante de su política cultural. Su objetivo no es solo brindar información sobre el valor cultural de los expertos al público, sino también incluir a dicho público en los proyectos de protección y uso de dicho patrimonio en aras de formar la identidad o para promover el desarrollo económico de la zona. La idea principal es que el patrimonio cultural, perteneciente anteriormente solo al mundo académico, se comparte con el público. Por tal motivo, se requiere la participación de la comunidad local en los programas de conservación y uso social del patrimonio cultural. Sin embargo, existe una relación asimétrica o vertical entre los expertos y el público debido a que se necesita de conocimientos y técnicas particulares para entender el valor del patrimonio cultural, especialmente del patrimonio arqueológico. Para modificar esta relación se debe prestar atención no solamente al valor científico del patrimonio, sino también al patrimonio cultural inmaterial de las comunidades locales dado que, en el caso del manejo del patrimonio cultural inmaterial, estas comunidades pueden tomar la iniciativa. En este artículo presentaré las actividades que el Proyecto Pacopampa viene realizando en una comunidad ubicada en la sierra norte del Perú, tratando de encontrar una forma de conectar los usos entre el patrimonio cultural material e inmaterial para promover la participación de la comunidad local.
\end{abstract}

Palabras clave: patrimonio cultural, cultura material, cultura inmaterial, memoria social, participación de la comunidad local.

Abstract: Recently, Peru has taken a positive attitude regarding the enhancement of cultural
heritage as an important element of its cultural policy. Its objective is not only to provide
information on the cultural value of experts to the public, but also to include the public in projects
for the protection and use of the heritage in order to form identity or to promote the economic
development of the area. The main idea is that cultural heritage, previously belonging only to the
academic world, is shared with the public. For this reason, the participation of the local community
in the programs of conservation and social use of cultural heritage is required. However, there is
an asymmetric or vertical relationship between experts and the public because it takes particular
knowledge and techniques to understand the value of cultural heritage, especially archaeological
heritage. To modify this relationship, attention should be paid not only to the scientific value of
the heritage, but also to the intangible cultural heritage of local communities, given that, in the
case of the management of intangible cultural heritage, these communities can take the initiative.

Acta Hispanica, Hungría, Supplementum II: 737-746, 2020, ISSN: 1416-7263 |737 


\section{Relacionando el patrimonio cultural material e inmaterial para su uso y protección en la sierra norte del Perú}

In this article I will present the activities that the Pacopampa Project has been carrying out in a community located in the north highlands of Peru, trying to find a way to connect the uses between the material and intangible cultural heritage to promote the participation of the local community.

Keywords: Cultural Heritage, Tangible Heritage, Intangible Heritage, Social Memory, Local Community Participation.

\section{Introducción}

Actualmente los arqueólogos y expertos en patrimonio cultural prestan atención a la participación o incorporación de la comunidad circundante en los proyectos de conservación y el uso social de dicho patrimonio. Los recursos económicos son solo considerados en algunas circunstancias. Una razón para esta tendencia son los movimientos sociales de comunidades indígenas están empezando a exigir su derecho en la participación de tales proyectos, un reclamo que se ve notablemente en las reuniones de la UNESCO (Cobb and Fowler, 2007).

Otra razón es el auge del neoliberalismo económico. Si bien es aplicado de maneras distintas dependiendo del país, en todos los casos se busca políticas dependientes de la economía de mercado para lograr mejorar los gobiernos pequeños (Von Schnitzler, 2008). Al mismo tiempo, los neoliberalistas reclaman que los ciudadanos liberados del patrocinio del estado deben tener autonomía. Es así como el neoliberalismo se utiliza como una ideología para reforzar los recortes presupuestarios relacionados al patrimonio cultural (Seki, 2016: 105). La autonomía se dirige a la economía y se requiere esfuerzos aparte para conseguir fondos. Se puede decir que el desarrollo cultural y lo económico se unen en una línea directa. Esta situación también es básicamente aplicable en Perú, que es el tema de discusión en este artículo (Sheahan, 2006).

Aparte de estas razones, existe otro factor en la participación o incorporación de las comunidades en los proyectos de conservación y uso social del patrimonio cultural: la transformación del estudio de las ciencias sociales, en otras palabras, la influencia de los estudios postcoloniales y la reflexión sobre la antropología por parte de los antropólogos mismos (Clifford and Marcus, 1986). Anteriormente, los antropólogos ignoraban la formación de la cultura "tradicional" y la habían identificado como una cultura sólida y pura que no debe cambiar. Se preocupaban por la desaparición de la cultura tradicional debido a la modernización desde un punto de vista opuesto, indicando que se estaban violando los derechos de las comunidades, algo que requería un cambio. Las narraciones donde la cultura es transformada eran consideradas como una falsificación que despoja el derecho de las comunidades a creer en su propia autenticidad. En cambio, los postcolonialistas hablan ahora de que la decisión de si algo es falso o auténtico debe dejarse en manos de las comunidades, no de los antropólogos. En cualquier caso, es claro que narrar sobre la cultura ya no se limita a los investigadores. 
Yuji Seki - Daniel Saucedo Segami

Considerando las circunstancias mencionadas, es natural pensar que las comunidades deben participar en las decisiones política cultural. Sin embargo, con esto no desaparecen los conflictos sobre el patrimonio entre comunidades, arqueólogos e instituciones encargadas de los asuntos relacionados a la política cultural.

La situación se relaciona a la estructura de área del estudio. En caso del patrimonio intangible o inmaterial, como religión o danza tradicional, el portador está únicamente en el lado de la comunidad. Ciertamente, incluso si existe un conocimiento tácito que los investigadores notan, estos se encuentran generalmente están en una posición de observador. En ese sentido, la comunidad está mejor calificada que el investigador.

Sin embargo, en caso del patrimonio material, sobre todo el patrimonio arqueológico, la posición se invierte (Seki, 2017). Los investigadores, es decir, los arqueólogos, tienen mucho más conocimiento y habilidades que los miembros de la comunidad. Las excavaciones son difíciles de realizar al menos a que se tengan suficientes conocimientos y habilidades académicas. En ese sentido, una relación en la que el investigador destaca es clara, donde los investigadores o expertos relacionados al patrimonio arqueológico plantean varios programas, como la construcción de museos de sitio y su manejo administrativo, así como también en el área de educación. Si bien se debe reconocer el valor de estos esfuerzos, y no dudo que hay que promover dichos programas, es en los términos "educación" o "capacitación" que se observa una relación asimétrica o vertical entre los expertos y el público: los educadores y los educados. En esta circunstancia, los educadores o expertos a veces o frecuentemente toman la iniciativa para poner en valor al patrimonio cultural sin escuchar las voces de los educados o del público.

La pregunta que surge entonces es si solo los expertos pueden poner en valor al patrimonio cultural y si el público debe solo gozar de los beneficios de la información obtenida. En esta ponencia presentaré una alternativa sobre la puesta en valor del patrimonio cultural que incluye la activa participación del público.

El punto clave de este tema es la memoria social, la cual es definida como una memoria colectiva, no personal, la cual es mantenida por un grupo o comunidad mediante actividades repetitivas (Van Dyke and Alcock, 2003: 2). Presentaré aquí un caso de la sierra norte del Perú.

\section{Aparición de la memoria social en Pacopampa}

El equipo de estudio conjunto de Japón y Perú, dirigido por el autor, ha estado excavando el sitio arqueológico Pacopampa desde 2005. El sitio se localiza en el centro poblado Pacopampa, distrito de Querocoto, provincia de Chota, Región Cajamarca; a 2500 metros sobre el nivel del mar en la vertiente oriental de la cordillera occidental de los Andes (Fig. 1).

Acta Hispanica, Hungría, Supplementum II: 737-746, 2020, ISSN: 1416-7263 |739 
Relacionando el patrimonio cultural material e inmaterial para su uso y protección en la sierra norte del Perú

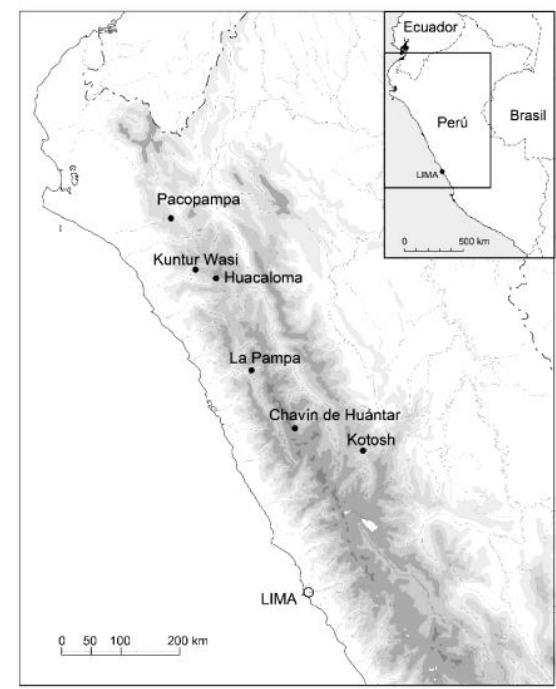

Fig. 1 Ubicación de los sitios arqueológicos mencionados en el artículo

Está compuesto de tres plataformas grandes. Las construcciones principales se encuentran en la Tercera Plataforma, es decir la plataforma superior (Fig. 2).

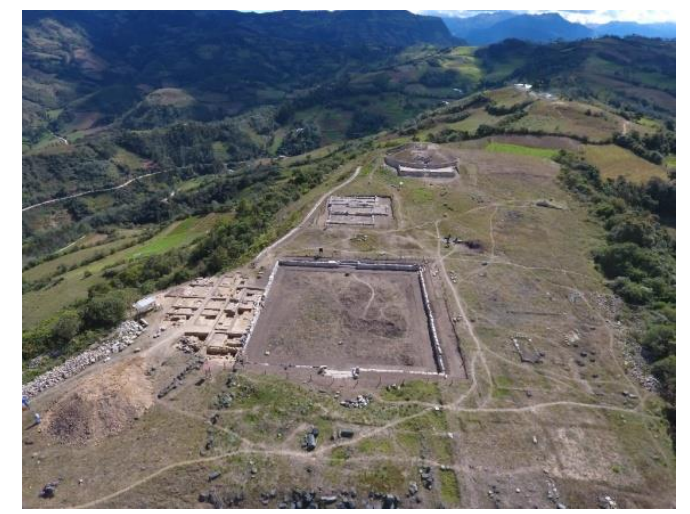

Fig. 2 Vista general del sitio Arqueológico Pacopampa CHeinz Plenge

Si bien ha sido investigado anteriormente por varios arqueólogos, quienes han presentado sus propias cronologías, nuestro Proyecto Arqueológico Pacopampa UNMSM-MNE, a partir de datos estratigráficos y de materiales asociados recuperados, ha establecido principalmente dos fases (Seki - Inokuchi y Morales 2010): Pacopampa I (1200 a.C. - 700 a.C.) y Pacopampa II (800 a.C.- 400 a.C.).

Cuando se observa el pie desde la cresta donde se encuentra el sitio arqueológico de Pacopampa, se puede ver un pequeño pueblo compuesto por casi 500 familias (Fig. 3). 


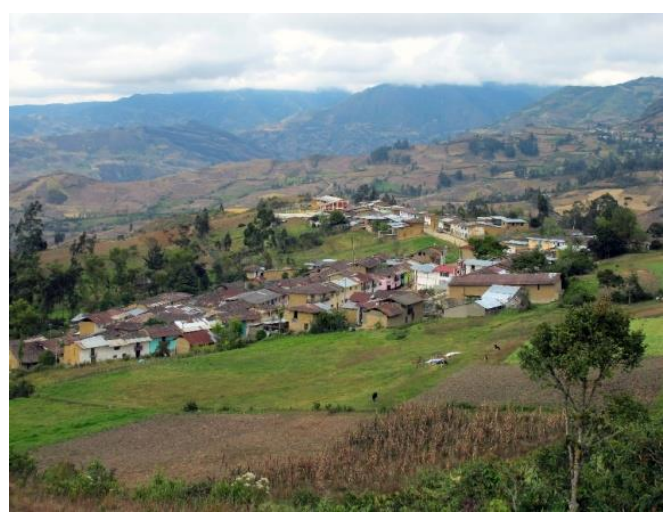

Fig. 3 Pueblo Pacopampa

Es un pueblo con el mismo nombre que el sitio arqueológico y se dice que el pueblo fue fundado hace unos 150 años (Cueva, 1982: 10). Políticamente, pertenece a la categoría de Centro Poblado (pueblo) que se encuentra bajo el Distrito, Provincia y Región. El jefe del pueblo existe, pero es solo un puesto honorario.

Durante estos trece años de investigación, siempre he tenido en cuenta la colaboración con la comunidad a través de reuniones de explicación del trabajo y exposiciones temporales de las piezas recuperadas en las excavaciones (Seki, 2017). Además, hemos encargado a la comunidad la selección y el salario de los trabajadores del proyecto. Este esfuerzo surtió efecto y la Asociación Cultural de Pacopampa fue fundada voluntariamente para conservar el sitio arqueológico en el año 2012. El motivo de la fundación de la asociación fue el descubrimiento de tumbas con ofrendas suntuosas (Fig. 4).

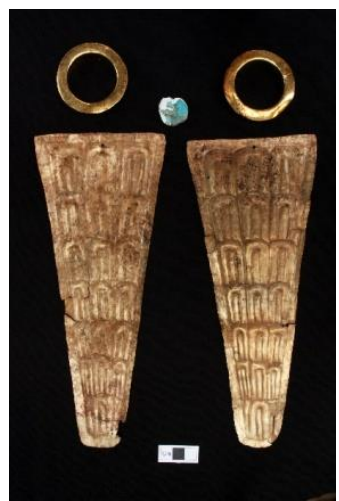

Fig. 4 Ofrendas de oro asociadas a la tumba de Dama de Pacopampa 


\section{Relacionando el patrimonio cultural material e inmaterial}

para su uso y protección en la sierra norte del Perú

La tumba fue nombrada la tumba de la Dama de Pacopampa. Al igual que Kuntur Wasi, un sitio en la misma región donde la Misión Arqueológica Japonesa apoyó para la construcción del primer museo de sitio comunal en el Perú (Onuki, 2006; Seki, 2016), la comunidad de Pacopampa desea tener un museo del sitio y actualmente su proyecto de construcción está en camino.

En Pacopampa podemos ver el surgimiento o la manifestación de la memoria social en la forma de la fiesta patronal del pueblo (Seki, 2017). El 30 de agosto es el día central de la fiesta dedicada a Santa Rosa. El programa de la fiesta contiene varias actividades como partidos de fútbol, corrida de toros, elección de Miss Pacopampa, feria de productos agropecuarios, y baile además de actividades religiosas. Gracias a la idea propuesta por el comité organizador de la fiesta, se agregó un desfile de disfraces interpretado por niños y jóvenes desde el año 2015. En el desfile de los niños de jardín se encuentran no solamente de Jesucristo y Santa Rosa, sino también la Dama de Pacopampa, el personaje enterrado en la tumba asociada a las ofrendas de oro que nuestro proyecto encontró. La niña que representaba a la Dama de Pacopampa era acompañada por una mujer adulta, posiblemente la mamá de niña, quien tenía un cartel donde estaba escrito "Pacopampa Capital Arqueológica”. Después de ellos, apareció un niño con sombrero y bastón con las mangas recogidas y con una faja llevando escrito mi nombre. Le seguían las niñas disfrazadas de dos miembros japoneses del proyecto. Por otro lado, en el desfile de los estudiantes de la escuela primaria se veía la historia del mundo mezclada con la historia local. Comenzando desde la época de cazadores, le seguían la Dama de Pacopampa (Fig. 5), los Incas, la época colonial, los primeros sacerdotes cristianos del pueblo, el alcalde, el fiscal y nuevamente Yuji Seki. Nosotros no hemos aconsejado sobre esta actividad ni hemos dado información a los organizadores del evento. La existencia de nosotros o los resultados de las investigaciones están incorporados en la conciencia histórica de la comunidad por su propia iniciativa.

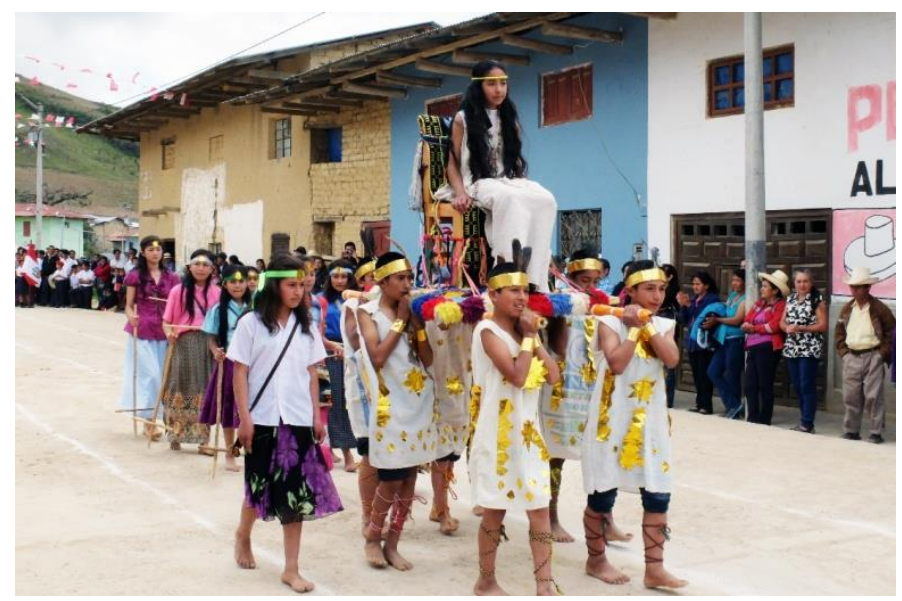

Fig. 5 Desfile de los estudiantes quienes cargan la Dama de Pacopampa 
La memoria social siempre nace o se forma y se fija a través de la repetición de las actividades, pero no siempre nace por intervención directa de los arqueólogos (Seki, 2017). Si las actividades de los arqueólogos motivan la formación de la memoria social, la memoria aparece por accidente. Lo importante es que los arqueólogos se den cuenta de esto y fomenten la creación de esta memoria en el contexto del uso social del patrimonio, en vez de negarla. A través del tratamiento de la memoria social, podemos establecer una mejor relación entre los arqueólogos y la comunidad.

No obstante, hay que prestar atención a nuestra posición en el desfile. La comunidad seleccionó los santos, héroes y las autoridades del pueblo para esta actividad, lo cual quiere decir que nosotros los arqueólogos también estamos situados en el mismo contexto. Para la comunidad, nosotros somos vistos como los dirigentes y las autoridades del pueblo. Aunque la memoria social surgió por voluntad propia del pueblo, se refleja una relación de poder también. En este sentido, incluso con énfasis en las memorias sociales únicas de los pobladores, las relaciones verticales que existen entre los investigadores y los pobladores solo se corrigen parcialmente.

\section{Involucrando el patrimonio inmaterial como memoria social en el proyecto del uso social del patrimonio}

Nos centraremos en el patrimonio inmaterial de los pobladores. Como he dicho anteriormente, en el caso del patrimonio intangible o inmaterial, como costumbres religiosas o danzas tradicionales, el portador está únicamente en el lado de la comunidad. Además, el patrimonio inmaterial es una clase de memoria social debido a que se forma por actividades repetitivas en la vida cotidiana o religiosa. Por lo tanto, al combinar el patrimonio intangible con el patrimonio material mencionado anteriormente, la relación vertical entre los investigadores y los pobladores puede verse disminuida o compensada.

Con tal perspectiva, desde el año 2017 estamos organizando un taller en el pueblo de Pacopampa para establecer un banco de datos del patrimonio inmaterial de su gente. El título del taller se llama "Buscando los tesoros". El motivo principal del taller es que los pobladores mismos reconozcan el valor del patrimonio inmaterial que se encuentran en el pueblo, pero que no llegan a identificar.

Como primera etapa, el 2017 empezamos preguntando a los pobladores en múltiples reuniones sobre los recursos culturales representativos del pueblo y cuales querían mostrar a los forasteros. Cada participante del taller escribió un recurso cultural en una nota adhesiva, luego nosotros las juntamos y clasificamos junto con ellos en categorías y subcategorías. Al final, pudimos recuperar un total de 216 recursos, divididos en cuatro grandes categorías y 16 subcategorías.

En el siguiente año 2018, les pedimos a los pobladores que eligieran una subcategoría favorita y que ordenen los recursos de acuerdo con el grado de mayor importancia para ellos. De acuerdo con su interés en cada subcategoría, se formaron grupos y se eligió a un coordinador. Solicitamos luego a cada grupo que describan las características de cada 


\section{Relacionando el patrimonio cultural material e inmaterial}

para su uso y protección en la sierra norte del Perú

recurso en una ficha hecha por nosotros, especificando detalles sobre cuándo, dónde, quién, y cómo se utiliza cada recurso. Se les motivó, además, a realizar dibujos o adjuntar información extra como dibujos, fotografías, etc. Al terminar, cada ficha fue digitalizada inmediatamente (Fig. 6).

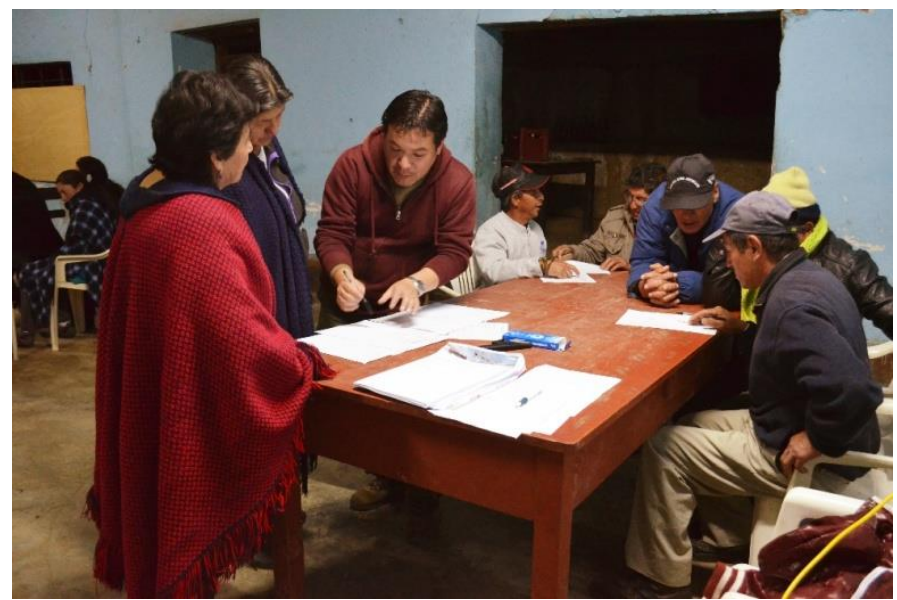

Fig. 6 Taller para recuperar informaciones de las culturas inmateriales del pueblo

Al final del taller se presentaron los resultados de la priorización y los contenidos de los recursos basados en ella. Sorprendentemente, algunos grupos realizaron entrevistas con los ancianos del pueblo durante los talleres para obtener más información sobre sus recursos.

Paralelamente al taller, llevamos a cabo el trabajo de tomar fotografías y videos de los recursos seleccionados por los pobladores. Además, realizamos la digitalización de fotografías antiguas que varias familias tenían con miras a formar un archivo fotográfico. Estas fotografías a menudo muestran los recursos elegidos por los aldeanos en diferentes momentos de la historia del pueblo. En el taller, estas fotos digitalizadas fueron revisadas por los pobladores y se recogieron sus comentarios. De esta manera, estamos construyendo la base de datos sobre el patrimonio intangible o inmaterial existente en el pueblo con su misma gente.

Hay múltiples propósitos para construir la base de datos. Uno es para usarla en la exhibición del Centro de Interpretación cuya construcción se encuentra actualmente en trámite con el Gobierno Regional. El diseño de la implementación del centro ya se ha completado y, según el mismo, tendrá al menos cuatro espacios de exhibición. Además de proporcionar información arqueológica sobre las investigaciones de Pacopampa, también proporcionará información sobre el patrimonio inmaterial del pueblo como son su historia, costumbres, tradiciones y forma de vida. La base de datos se podrá utilizar para determinar el contenido de dichas exhibiciones, un trabajo que naturalmente se realizará con los pobladores. 
La exhibición del patrimonio inmaterial no solo servirá para que los pobladores reconozcan sus contenidos y valores, sino que también servirá para preparar el entorno donde ellos participen voluntariamente de la operación del Centro de Interpretación en el futuro. Al crear el espacio donde ellos tengan la oportunidad de transmitir sus conocimientos sobre el patrimonio inmaterial, se promoverá a su vez esta participación voluntaria.

El Centro de Interpretación no pretende acumular y presentar físicamente todo el patrimonio inmaterial del pueblo. Más bien, este espacio servirá como un punto donde los visitantes podrán acceder a información introductoria que les permita recorrer y experimentar el patrimonio inmaterial en el pueblo. Es decir, se pretende que este centro funcione bajo el concepto de Ecomuseo, de manera tal que no solo quienes operen el centro participaran en la preservación y uso del patrimonio cultural, sino toda la población.

La base de datos también contribuirá a la educación en los colegios del pueblo. Planeamos utilizar la base de datos para diseñar un programa que permita a los estudiantes acercarse, conocer, examinar y experimentar el patrimonio inmaterial del pueblo. Como resultado, se logrará que la información sobre el patrimonio inmaterial, que está perdiendo, pase a la siguiente generación y se cree así una identidad local, preparando a la vez la base para utilizarlo como recurso turístico.

De esta manera, el significado y valor del patrimonio tangible proporcionado por los investigadores se combina con la memoria social sobre el patrimonio material de los pueblos, así como también con otra clase de memoria social que los pobladores tienen de manera autodirigida, es decir, el patrimonio inmaterial. Creemos que será posible preservar y utilizar integralmente el patrimonio cultural del pueblo. Sin embargo, aún es un camino con muchas dificultades, por lo que esperamos poder avanzar poco a poco, verificando cada paso que damos.

\section{Referencias bibliográficas}

Cobb, Daniel M. - Loretta, Fowler (2007). Beyond Red Power: American Indian Politics and Activism since 1900. Santa Fe: School for Advanced Research Press.

Clifford, James - Marcus, George E. (1986). Writing Culture: The Poetics and Politics of Ethnography. Los Angeles: University of California Press.

Cueva, Nicanor Alarcón (1982). Pacopampa. Lima: Seminario de Historia Rural Andina, Universidad Nacional Mayor de San Marcos.

Onuki, Yoshio (2006). The Kuntur Wasi Museum in Northern Peru. En: Silverman, Helaine (ed.). Archaeological Site Museum in Latin America. Gainesville: University of Florida. 64-71. 
Relacionando el patrimonio cultural material e inmaterial para su uso y protección en la sierra norte del Perú

Seki, Yuji (2017). Formation of a Community Based on the Cultural Heritage: A Case from the South America. En: Iida, Taku (ed.). Cultural Heritage in the Civilization. Kyoto: Rinsen Shoten. 63-93. (in Japanese).

Seki, Yuji (2016). Participation of the Local Community in Archaeological Heritage Management in the North Highlands of Peru. En: Underhill, Anne P. - Salazar, Lucy C. (eds.). Finding Solutions for Protecting and Sharing Archaeological Heritage Resources. Heidelberg: Springer. 103-119.

Seki, Yuji - Villanueva, Juan Pablo - Sakai, Masato - Alemán, Diana - Ordoñez, Mauro - Tosso, Walter - Espinoza, Araceli - Inokuchi, Kinya - Morales, Daniel (2010). Nuevas evidencias del sitio arqueológico de Pacopampa, en la sierra norte del Perú. Boletín de Arqueología PUCP, 12. 69-95.

Sheahan, John (2006). Redirection of Peruvian Economic Strategy in the 1990s: Gains, Losses, and Clues for the Future. En: Carrión, Julio F. (ed.). The Fujimori Legacy: The Rise of Electoral Authoritarianism in Peru. University Park, PA: The Pennsylvania State University Press. 178-200.

Van Dyke, Ruth M. - Alcock, Susan E. (2003). Archaeology of Memory: An Introduction. En: Van Dyke, Ruth M. - Alcock, Suan E. (eds.). Archaeologies of Memory. Malden: Blackwell Publishers Ltd. 1-13.

Von Schnitzler, Antina (2008). Neoliberalism. En: Darity, William A.(ed.). International Encyclopedia of the Social Science, 2nd edition. Detroit, New York, San Francisco, New Haven, Conn, Waterville, Maine, London: The Gale Group. 473-475. 